\title{
The EMBARC European Bronchiectasis Registry: protocol for an international observational study
}

\author{
James D. Chalmers ${ }^{1,33}$, Stefano Aliberti2,33, Eva Polverino ${ }^{3,33}$, \\ Montserrat Vendrell ${ }^{4}$, Megan Crichton ${ }^{1}$, Michael Loebinger ${ }^{5}$, \\ Katerina Dimakou 6 , Ian Clifton 7 , Menno van der Eerden ${ }^{8}$, Gernot Rohde 9 \\ Marlene Murris-Espin ${ }^{10}$, Sarah Masefield ${ }^{11}$, Eleanor Gerada ${ }^{12}$, \\ Michal Shteinberg ${ }^{13}$, Felix Ringshausen ${ }^{14}$, Charles Haworth ${ }^{15}$, Wim Boersma ${ }^{16}$, \\ Jessica Rademacher ${ }^{14}$, Adam T. Hill ${ }^{17}$, Timothy Aksamit ${ }^{18}$, Anne O'Donnell ${ }^{19}$, \\ Lucy Morgan ${ }^{20}$, Branislava Milenkovic ${ }^{21,22}$, Leandro Tramma ${ }^{1}, J_{0 a o}$ Neves $^{23}$, \\ Rosario Menendez ${ }^{24}$, Perluigi Paggiaro ${ }^{25}$, Victor Botnaru ${ }^{26}$, Sabina Skrgat ${ }^{27}$, \\ Robert Wilson ${ }^{5}$, Pieter Goeminne 28 , Anthony De Soyza 29,30 , Tobias Welte ${ }^{14}$, \\ Antoni Torres ${ }^{3}$, J. Stuart Elborn ${ }^{31}$ and Francesco Blasi ${ }^{32}$, on behalf of EMBARC.
}

Affiliations: ${ }^{1}$ College of Medicine, University of Dundee, Ninewells Hospital and Medical School, Dundee, UK. ${ }^{2}$ Dept of Health Science, University of Milan Bicocca, Clinica Pneumologica, AO San Gerardo, Monza, Italy. ${ }^{3}$ Fundaciò Clìnic, IDIBAPS, CIBERES, Hospital Clinic de Barcelona, Barcelona, Spain. ${ }^{4}$ Bronchiectasis Group, Girona Biomedical Research Institute (IDIBGI), Dr Trueta University Hospital, Girona, Spain. ${ }^{5}$ Host Defence Unit, Royal Brompton Hospital, London, UK. '5th Pulmonary Department, "Sotiria" Chest Hospital, Athens, Greece. ${ }^{7}$ Leeds Centre for Respiratory Medicine, St James's Hospital, Leeds, UK. ${ }^{8}$ Dept of Pulmonary Medicine, Erasmus MC Rotterdam, Rotterdam, The Netherlands. ${ }^{9}$ Dept of Respiratory Medicine, Maastricht University Medical Center, Maastricht, The Netherlands. ${ }^{10}$ Pôle des Voies Respiratoires, Hôpital de Larrey, $\mathrm{CHU}$ de Toulouse, Toulouse, France. ${ }^{11}$ European Lung Foundation, Sheffield, UK. ${ }^{12}$ Dept of Respiratory Medicine, Mater Dei Hospital, Msida, Malta. ${ }^{13}$ Pulmonary Institute, Carmel Medical Center, Haifa, Israel. ${ }^{14}$ Dept of Respiratory Medicine, Hannover Medical School, Member of the German Centre for Lung Research, Hannover, Germany. ${ }^{15}$ Cambridge Centre for Lung Infection, Papworth Hospital, Cambridge, UK. ${ }^{16}$ Dept of Pulmonary Diseases, Medical Centre Alkmaar, Alkmaar, The Netherlands. ${ }^{17}$ Royal Infirmary of Edinburgh, University of Edinburgh, Edinburgh, UK. ${ }^{18}$ Mayo Clinic College of Medicine, Rochester, MN, USA. ${ }^{19}$ Division of Pulmonary, Critical Care and Sleep Medicine, Georgetown University Medical Center, Washington, DC, USA. ${ }^{20}$ Dept of Respiratory Medicine, Concord Hospital, Concord Clinical School, University of Sydney, Sydney, Australia. ${ }^{21}$ Faculty of Medicine, University of Belgrade, Belgrade, Serbia. ${ }^{22}$ Clinic for Pulmonary Diseases, Clinical Center of Serbia, Belgrade, Serbia. ${ }^{23}$ Medicina Interna, Centro Hospitalar do Porto, Porto, Portugal. ${ }^{24}$ Pneumology Service, Universitary and Polytechnic Hospital La Fe, Valencia, Spain. ${ }^{25}$ Dept of Surgery, Medicine, Molecular Biology and Critical Care, University of Pisa, Pisa, Italy. ${ }^{26}$ Division of Pneumology, Dept of Internal Medicine, State Medical University of Medicine and Pharmacy "Nicolae Testemitanu", Chisinau, Republic of Moldova. ${ }^{27}$ University Clinic of Respiratory and Allergic Diseases Golnik, Golnik, Slovenia. ${ }^{28}$ Dept of Respiratory Medicine, AZ Nikolaas, Sint-Niklaas, Belgium. ${ }^{29}$ Institute of Cellular Medicine, Newcastle University, Newcastle upon Tyne, UK. ${ }^{30}$ Bronchiectasis Service, Freeman Hospital, Newcastle upon Tyne, UK. ${ }^{31}$ School of Medicine, Dentistry and Biomedical Sciences, Queen's University of Belfast, Belfast, UK. ${ }^{32}$ Respiratory Unit, IRCCS Fondazione Cà Granda Milano, Dept of Pathophysiology and Transplantation, University of Milan, Milan, Italy. ${ }^{33}$ These authors contributed equally.

Correspondence: James D. Chalmers, College of Medicine, University of Dundee, Ninewells Hospital and Medical School, Dundee, DD1 9SY, UK. E-mail: jchalmersdadundee.ac.uk

Received: Oct 292015 | Accepted: Nov 022015 | First published: Jan 202016

This article has been republished to update reference 22 .

This article has supplementary material available from openres.ersjournals.com

Support statement: The European Multicentre Bronchiectasis Audit and Research Collaboration is a European Respiratory Society Clinical Research Collaboration and is part of the iABC consortium supported by the European Union Innovative Medicines Initiative. EMBARC has also received funding and support from the US COPD Foundation, Bayer HealthCare and Aradigm Corporation. Funding information for this article has been deposited with FundRef.

Conflict of interest: S. Masefield is an employee of the European Respiratory Society. Disclosures can be found alongside this article at openres.ersjournals.com

The content of this work is @ the authors or their employers. Design and branding are OERS 2015. This article is open access and distributed under the terms of the Creative Commons Attribution Non-Commercial Licence 4.0. 
ABSTRACT Bronchiectasis is one of the most neglected diseases in respiratory medicine. There are no approved therapies and few large-scale, representative epidemiological studies.

The EMBARC (European Multicentre Bronchiectasis Audit and Research Collaboration) registry is a prospective, pan-European observational study of patients with bronchiectasis. The inclusion criterion is a primary clinical diagnosis of bronchiectasis consisting of: 1) a clinical history consistent with bronchiectasis; and 2) computed tomography demonstrating bronchiectasis. Core exclusion criteria are: 1) bronchiectasis due to known cystic fibrosis; 2) age <18 years; and 3) patients who are unable or unwilling to provide informed consent.

The study aims to enrol 1000 patients by April 2016 across at least 20 European countries, and 10000 patients by March 2020. Patients will undergo a comprehensive baseline assessment and will be followed up annually for up to 5 years with the goal of providing high-quality longitudinal data on outcomes, treatment patterns and quality of life. Data from the registry will be available in the form of annual reports. and will be disseminated in conference presentations and peer-reviewed publications.

The European Bronchiectasis Registry aims to make a major contribution to understanding the natural history of the disease, as well as guiding evidence-based decision making and facilitating large randomised controlled trials.

$@$ ERSpublications

The European Bronchiectasis Registry will recruit 10000 patients over 5 years http://ow.ly/Ul7Pd

\section{Introduction}

Bronchiectasis has historically been an under-researched and under-resourced condition, sometimes described as an "orphan disease" $[1,2]$. There has been minimal research into bronchiectasis over the last decades in contrast to other areas such as chronic obstructive pulmonary disease (COPD) and cystic fibrosis [3-5]. Unfortunately, the treatment of bronchiectasis remains largely based on expert opinion, unsupported by high-quality evidence [6-8]. Much of our current practice is extrapolated from COPD, cystic fibrosis or other respiratory infections.

The prevalence of bronchiectasis is significantly higher than the European Medicines Agency definition of an orphan disease (five cases in 10000). Data from the UK show a prevalence of 125.7 cases per 100000 person-years in 2013, a rate that has almost doubled in a decade from 2004 [9]. Data from Germany show a prevalence of 67 per 100000 [10]. These differences are likely to reflect underlying differences in how healthcare systems collect administrative data, as well as physician awareness of the disease. Hospital admissions and mortality from bronchiectasis are also rising in Europe and in the USA [11-13]. Therefore, bronchiectasis is placing an increasing burden on healthcare systems internationally, suggesting an urgent need for better treatments and better clinical care, and for clinical and translational research into this condition [14].

Recent years have seen a welcome increase in interest in the disease, described as "the age of bronchiectasis", but despite this, there have been few longitudinal or cross-sectional studies in well characterised populations of patients with bronchiectasis [15]. The heterogeneity of the disease, and its different epidemiology across Europe and various parts of the world, make single-centre or single-country studies hard to generalise [16, 17]. This has translated into difficulties in the development of new treatments, as a number of bronchiectasis clinical trials have failed to meet their primary end-point, failed to recruit patients to target or have been unable to define the optimal target population [18-21]. More data on the epidemiology of the disease are needed, but to give truly meaningful and generalisable results, studies need to enrol several thousand patients encompassing the aetiological, clinical, microbiological and international heterogeneity of the condition.

The European Respiratory Society (ERS) contributes to the coordination of activities in respiratory medicine across Europe through funding of Clinical Research Collaborations (CRCs), pan-European, multicentre networks of principal investigators aiming to advance science and clinical research within a specific disease area (www.ersnet.org/ers-funding/clinical-research-collaboration.html). The European Multicentre Bronchiectasis Audit and Research Collaboration (EMBARC) was established as an ERS CRC with the objective of developing a European Bronchiectasis Registry and bringing researchers together internationally to drive improvements in clinical research, clinical care and education. This article describes the protocol for the EMBARC European Bronchiectasis Registry, an open, pan-European registry of patients with clinically significant bronchiectasis.

\section{Study objectives}

The objectives of the study are:

- to develop a pan-European, multicentre bronchiectasis registry incorporating baseline data collection with annual follow-up data for at least 5 years; 
- to describe the demographics, comorbidities, aetiology, medication usage, resource consumption, exacerbations, microbiology, severity and prognosis of bronchiectasis across Europe (in summary, a comprehensive description of characteristics and burden of this disease across the continent);

- to facilitate multinational cooperation, within and outwith Europe; and

- to facilitate the creation of national registries in European countries that currently do not have a bronchiectasis research infrastructure.

The project as a whole also aims to ensure the needs and experiences of people with bronchiectasis across Europe inform all EMBARC activities. This has been achieved through the creation of a patient advisory group (PAG) and inclusion of PAG representatives on the EMBARC steering group. The EMBARC network is an open collaboration: anyone with an interest in bronchiectasis can join and take part in network activities.

\section{Methods and design of the EMBARC registry} Study design

The registry is a multicentre, prospective, observational cohort study enrolling consecutive adult patients with bronchiectasis across Europe. The study is noninterventional. Patients are managed according to their local procedures and policies with no interference from the study team. Patients give informed consent to participate and data are collected at baseline (recruitment) as described below. This includes comprehensive data across the following domains: demographics; comorbidities; aetiological testing; lung function; exacerbations; disease impact; quality of life (QoL); microbiology; radiology; and treatment and physiotherapy.

The study is set in primary and secondary care settings reflecting differences in how bronchiectasis is managed in different European countries. The study received central ethical approval from the Multicentre Research Ethics Committee in the UK on January 8, 2015 (14/SS/1101) and the study is sponsored by the University of Dundee, Dundee, UK. The study website is located at www.bronchiectasis.eu. The registry was developed in accordance with recommendations on the design, implementation, governance and long-term sustainability of disease registries in the European Union (EU), as proposed by the EPIRARE (European Platform for Rare Disease Registries) consortium and EUCERD [22, 23].

\section{Participants}

Patients should have a primary diagnosis of bronchiectasis, and meet the following inclusion and exclusion criteria. The inclusion criteria of this study are:

- a clinical history consistent with bronchiectasis (cough, chronic sputum production and/or recurrent respiratory infections); and

- computed tomography of chest demonstrating bronchiectasis (bronchial dilatation) affecting one or more lobes.

The exclusion criteria are:

- bronchiectasis due to known cystic fibrosis;

- age $<18$ years; and

- patients who are unable or unwilling to provide informed consent.

The exclusion criteria are deliberately sparse and the inclusion criteria broad to allow inclusion of a large and varied population representative of real-life bronchiectasis management across Europe.

Radiological bronchiectasis may be a common finding in the asymptomatic elderly or may be an incidental finding in other respiratory disorders such as COPD [24-26]. For the avoidance of doubt, patients with radiological bronchiectasis but without clinical symptoms and a physician diagnosis of clinically significant bronchiectasis will not be included in the study. This includes exclusion of patients with radiological bronchiectasis due to traction in interstitial lung disease where there is not a clinical diagnosis of bronchiectasis. The study also does not include patients who have undergone lung or heart-lung transplantation.

\section{Identification and recruitment}

Patients may be identified and recruited from outpatient clinics, specialist hospital clinics or in a primary care setting. An online survey conducted prior to the start of the study ( $n=282$ hospitals) suggested that patients with bronchiectasis are managed in a number of different settings. $52 \%$ of centres had a specialist bronchiectasis clinic, while $9.7 \%$ managed patients within a cystic fibrosis service. The remaining centres managed their patients in a general respiratory outpatient clinic. The study will include patients in all of these settings as the objective is to get a representative picture of bronchiectasis management and epidemiology in Europe. 


\section{Follow-up}

Study participants will then enter follow-up data for patients on an annual basis ( \pm 3 months) to give longitudinal data on changes in medication, exacerbation frequency, lung function, hospital admissions and survival data (figure 1). Linkage to electronic health records with patient consent will also be used where such resources are available at a national level.

\section{Data collection, variables and definitions}

Data will be collected in three distinct fields. 1) Baseline study entry: the basic dataset for recruitment of patients into the study. 2) Annual review form: follow-up data completed for each year of the study, indicating yearly exacerbation frequency, hospitalisations and survival status. 3) There is also an exacerbation substudy being conducted in a small number of sites collecting detailed information at the time of an acute exacerbation. A complete listing of registry fields is contained in the supplementary material.

The data fields have been developed and agreed following an exhaustive consultation process with stakeholders throughout Europe and in collaboration with the US and Australian registries [27]. The dataset will allow a number of ancillary studies to be proposed by investigators. Participants will be asked, on an optional basis, to provide consent for future contact with regard to taking part in clinical trials and studies. The network is intended to serve as a platform to engage researchers and facilitate collaborations around Europe. A screenshot of the data collection platform is shown in figure 2.

\section{Quality of life}

The registry uses the "Quality of Life - Bronchiectasis" questionnaire version 3.1 [28]. An automatic calculator tool is incorporated into the registry platform to aid calculation. This is used as it is the only disease-specific QoL tool that has been validated for bronchiectasis. It was developed using US Food and Drug Administration guidance on patient-reported outcomes, and is separated into multiple domains including physical functioning, role functioning, vitality, emotional functioning, social functioning, treatment burden, health perceptions and respiratory symptoms. Translations have been made available in 30 languages (www.psy.miami.edu/qol_b/translations.phtml) [27].

\section{Exacerbations}

The definition of exacerbation is debated and therefore, for pragmatic reasons, the registry defines exacerbation as an acute deterioration in the patient's condition resulting in a physician diagnosis of exacerbation and the prescription of systemic antibiotics [6]. Severe exacerbations will be defined by British Thoracic Society criteria as an exacerbation requiring hospital admission [6]. The database will also capture emergency department visits for exacerbations that do not result in hospital admission [6].

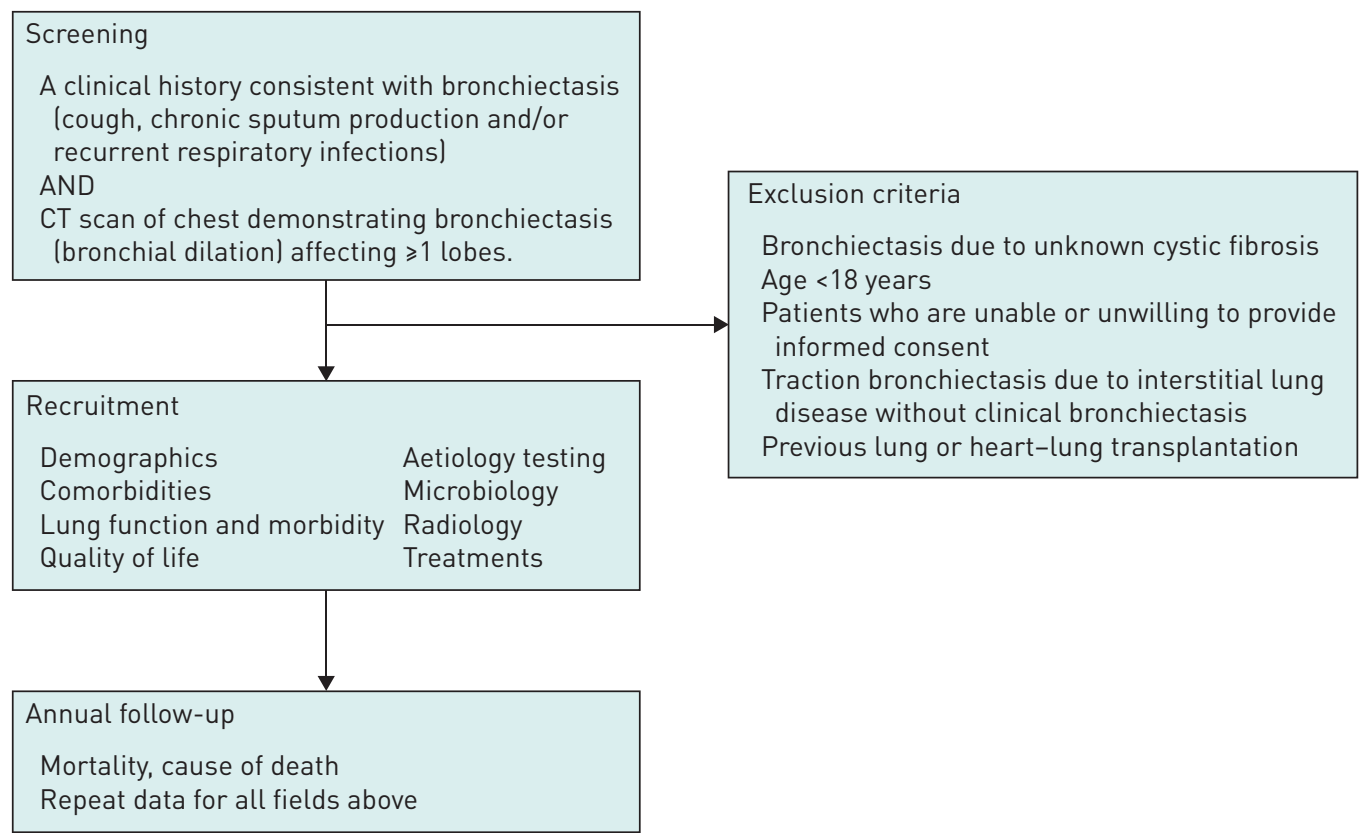

FIGURE 1 Study flowchart. CT: computed tomography. 


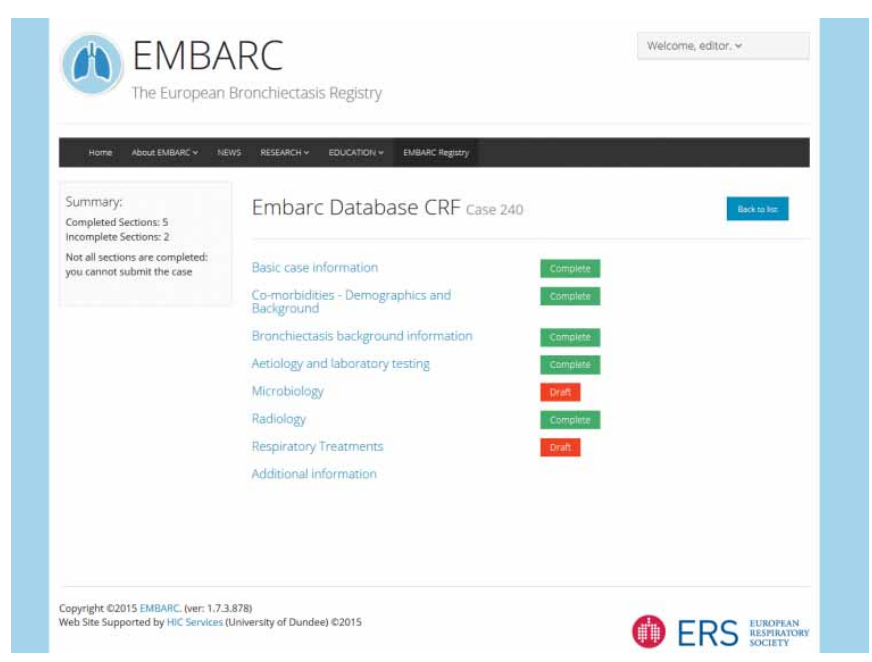

FIGURE 2 The European Multicentre Bronchiectasis Audit and Research Collaboration (EMBARC) data collection platform. Image courtesy of EMBARC and the Health Informatics Centre (University of Dundee, Dundee, UK).

\section{Sputum colour}

Sputum colour is evaluated using a validated, photographic sputum colour chart that rates colour from 1 (mucoid) to 4 (highly purulent) [29].

\section{Aetiology}

The aetiology of bronchiectasis is determined by the physician caring for the patient. Extensive data are collected on the aetiological testing performed, based on the testing recommended by consensus guidelines and, therefore, the underlying basis for an aetiological diagnosis is collected and can be validated [6].

\section{Lung function}

Raw values of height, weight, and post-bronchodilator forced expiratory volume in $1 \mathrm{~s}$ and forced vital capacity are recorded, allowing different equations for predicted values to be used as appropriate. Spirometry is performed according to American Thoracic Society/ERS standards [30].

\section{Severity of disease}

All of the variables required to calculate validated severity tools for bronchiectasis are recorded, such as history of hospital admissions, Medical Research Council dyspnoea score and radiological extent of disease. The database incorporates an automatic calculator for the Bronchiectasis Severity Index [17].

\section{Chronic obstructive pulmonary disease}

There is a reported association between COPD and bronchiectasis, but whether COPD is a cause of bronchiectasis and how to differentiate the two conditions is not clearly defined [31-33]. The dataset will capture both the physician diagnosis of COPD, and also the smoking history (current, ex- or never-smoker plus pack-years smoked) and the presence of airflow obstruction by spirometry, allowing a careful dissection of the relationships between these parameters.

\section{Microbiology}

Data are collected on any lower respiratory tract specimen (spontaneous, induced sputum or bronchoalveolar lavage) when clinically stable and during exacerbation [34]. A recent publication has highlighted different definitions of chronic colonisation but for the purposes of the study, colonisation is defined as two positive samples at least 3 months apart while clinically stable [34]. The database will also record antibiotic susceptibility testing. Pseudomonas aeruginosa has a strong association with morbidity and mortality that is consistent across multiple datasets [35-37]. Therefore, the database will collect specific data on the management of $P$. aeruginosa, which will include any historical isolation of $P$. aeruginosa, phenotype (mucoid versus nonmucoid) and the use of Pseudomonas eradication treatment.

\section{Radiology}

Complex radiological scoring is difficult to implement in an international multicentre registry. The modified Reiff score, which incorporates simple parameters of the degree of dilatation and the number of 
lobes involved, has been shown to correlate with mortality and hospital admissions in bronchiectasis, and is used in the study $[17,38]$.

\section{Treatments}

Details are recorded on a large number of treatment variables including all inhaled and oral therapies, antibiotics, mucoactive drugs and anti-inflammatories. Comprehensive data on physiotherapy practice is also recorded along with vaccination status. Treatment options will be regularly updated as new therapies complete randomised trials and become available during the course of the study.

\section{Quality control}

The database incorporates automated logic checks to prevent out-of-range values being entered or to feed back warnings to users when data are out of range. Each case entered into the registry is manually verified by a member of the study team and data queries are resolved with the study site. Cases with missing data or unresolved queries will be rejected to ensure only the highest quality data are retained in the registry. Source data verification and random audit will also be conducted at study sites.

\section{Sample size}

The sample has been determined empirically and set at a target of 8000-10000 patients by March 2020. A short-term target of 1000 patients in the first year of the project (up to April 2016) has been set. Representativeness is an important objective of the registry, and therefore we aim to include patients from a minimum of 20 European countries (figure 3). A clear objective of the registry is to make it sustainable over the long term. The study therefore aims to expand beyond these targets as resource permits.

\section{Governance, oversight and data sharing}

The registry is held securely in the University of Dundee Health Informatics Centre and anonymised data will be accessible to researchers throughout Europe, using the Safe Haven model (www.scot-ship-toolkit. org.uk/information-page/ship-safe-havens). This is a virtual desktop that allows secure data access and data analysis but prevents copying or downloading of data, thereby ensuring complete data security. Active investigators and other stakeholders will have unrestricted access to their own data. Requests to analyse the database as a whole will be managed by submission of a study protocol to the EMBARC scientific committee. The EMBARC registry is committed to open data sharing and the scientific committee operates on the basis that no reasonable request for data should be rejected. Full details regarding data access are reported on the EMBARC website (https://www.bronchiectasis.eu/dataaccess).

The database and governance processes surrounding data management and access are fully compliant with the UK Data Protection Act 1998 and the Data Protection Directive 95/46/EC of the European Parliament and of the Council (1995).

FIGURE 3 Distribution of EMBARC (European Multicentre Bronchiectasis Audit and Research Collaboration) sites across Europe.

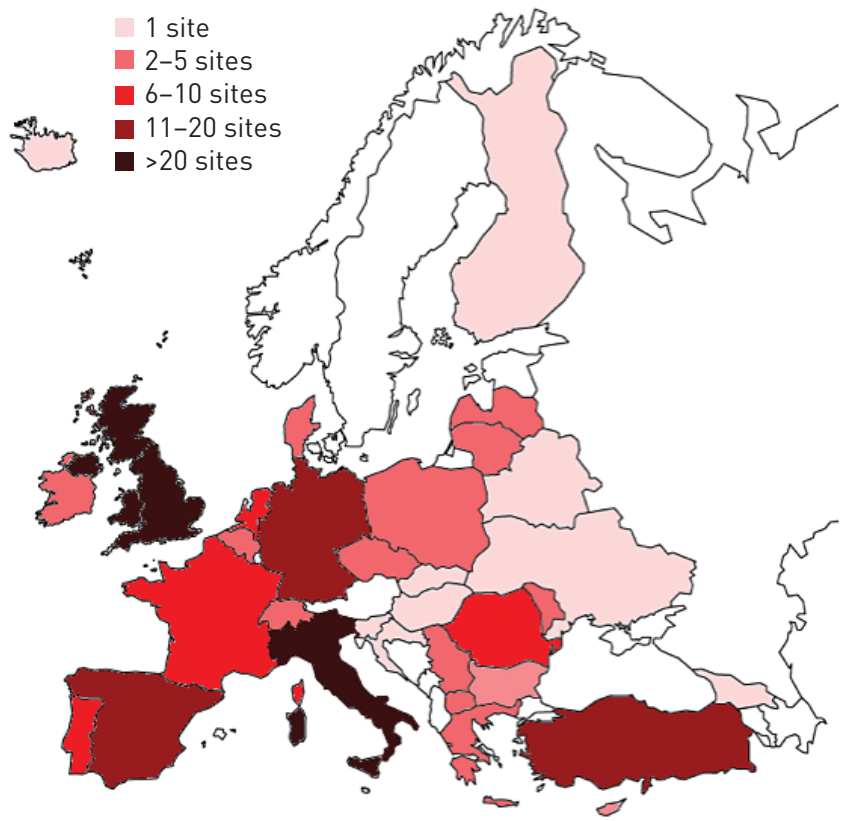


The study is conducted in accordance with the principles of Good Clinical Practice. A favourable ethical opinion is obtained by each partner site from the appropriate research ethics committee or institutional review board, where required. Additionally, any other necessary approvals required by partner sites are obtained prior to commencement of the study at each site. All patients must provide written informed consent to participate.

Study results will be disseminated in the form of annual reports, conference abstracts and peer-reviewed publications. The EMBARC study group will follow the International Committee of Medical Journal Editors recommendations regarding authorship.

\section{Patient involvement}

EMBARC is working closely with the European Lung Foundation (ELF) (www.europeanlung.org), which was founded by the ERS in 2000 with the aim of bringing together patients and the public with respiratory professionals to positively influence lung health. The design of the registry and EMBARC activities has been informed by review and feedback from patients and patient groups. ELF and EMBARC have ensured ongoing patient involvement in the network through the creation of a PAG comprised of people with bronchiectasis and those affected by bronchiectasis, such as a parent, partner or child of someone with bronchiectasis. There are currently 38 members across 10 European countries and ELF aims to recruit members from additional countries. Three members of this advisory group were selected to participate in the steering group of the bronchiectasis registry and will liaise with the group to ensure that the views of the group as a whole are represented (the job description for the steering committee role is included in the supplementary material). This approach embeds patients' views and priorities in the activities of the bronchiectasis network and the registry project.

A further outcome of this patient-professional collaboration will be to produce practical and reliable, medically accurate information resources in multiple languages via a codesigned patient website, and involve the patients in the development of planned educational resources.

\section{Future studies}

The EMBARC network has been developed to provide a platform for future international multicentre clinical trials and the first such trials are expected to begin in early 2016. In addition, the EMBARC platform has been developed to allow investigator-initiated ancillary studies and to support patient registries in countries outside Europe. EMBARC will make the registry platform available in Asia, Australasia and other continents to produce a harmonised dataset for international comparisons. The burden of bronchiectasis is not limited to adults, and future developments of the registry may seek to address bronchiectasis in children and/or to collect additional data in specific subgroups of patients such as those with nontuberculous mycobacterial (NTM) disease [39, 40]. The development of the EMBARC registry has benefitted greatly from collaborations with the US COPD Foundation Bronchiectasis and NTM registry and with the emerging registry in Australia.

In addition to its research focus, EMBARC will continue to promote multidisciplinary education and patient-professional collaboration in the field of bronchiectasis through its relationship with the ERS and ELF, with initiatives planned for 2016 to link to the publication of the ERS bronchiectasis guidelines.

\section{Discussion}

Disease registries have the potential to provide important insights into the natural history of disease, and to provide information required for health service planning, quality improvement and enrolment of patients into clinical trials [41].

Registries are essential in cases where diseases are sufficiently rare that epidemiological data cannot be provided by single-centre or even single-nation studies [42]. In developing the European Bronchiectasis Registry, we have followed guidance proposed in the EU and the extensive experience of the European Cystic Fibrosis Society Patient Register (ECFSPR), which has enrolled 30000 patients across the continent [42]. Recommendations from the ECFSPR have been published and have informed the development of the present project [42]. These recommendations include defining a minimum required dataset, limiting as far as possible the burden on participants entering the data. Standardising definitions across participating sites is essential to ensure that data are consistent and has been a challenge for the ECFSPR.

This has been resolved in the EMBARC registry through the use of a shared data collection platform and standardised definitions across all studies sites. National registries have fully collaborated with the European registry, sharing the data collection platform and protocols to ensure fully compatible data for collaborative analysis. The data collection tool has been made freely available to national registry groups to facilitate this. Data quality is being rigorously ensured. 
Bronchiectasis can no longer be regarded as a rare disease but remains neglected in the sense that there is a still a paucity of research data and there are no therapies licensed by regulatory authorities in Europe or the USA [41]. An improved understanding of the pathophysiology of bronchiectasis is needed in order to develop improved therapies, and registries can greatly enhance this kind of research by improving disease phenotyping and by linking to patient biobanks/bioresources [43, 44].

Epidemiological studies are challenging because bronchiectasis is both a radiological disorder and a clinical disease. Administrative databases can provide important information on disease burden but may greatly underestimate the true incidence where bronchiectasis is frequently not recognised or misdiagnosed as COPD, but might also overestimate the prevalence of the disease if the radiological disorder is recorded without also requiring a clinical syndrome of cough, sputum production and respiratory infections [9-14]. Registries therefore provide an important adjunct to such data by providing higher quality phenotyping while also providing data in countries where administrative data is not available $[45,46]$.

Late-phase clinical trials in bronchiectasis have struggled to meet their primary end-points in recent years for a variety of reasons [18-21]. In some cases, this may reflect differences in the pathophysiology of the disease but may also reflect a lack of data on patient stratification, the natural history of the condition or simply a lack of patients available for recruitment. It is hoped that the registry can facilitate clinical trials by aiding identification of patients, and by providing high-quality data on patient stratification and prognosis.

The ultimate objective of the European Bronchiectasis Registry should be to provide high-quality research that can contribute to evidence-based guidelines and have an impact on patient care, both in Europe and internationally. Linked to the EMBARC project, the ERS recently approved bronchiectasis guidelines to be published in 2016. These will be the first international guidelines for the disease, following the important contribution made by national guidelines in the UK and Spain, and recently in Australia and New Zealand [6-8]. To date, these guidelines have been largely formulated on the basis of expert opinion, and it should be a key objective of the registry to produce new evidence that can change clinical practice and guideline recommendations.

\section{Conclusion}

The European Bronchiectasis Registry aims to recruit 10000 patients across 20 European countries by 2020 and, therefore, to provide the largest and most detailed analysis of the natural history and impact of bronchiectasis to date. A sustainable registry promises to make an important contribution to improving the outlook for this neglected disease.

\section{Acknowledgements}

EMBARC acknowledgements partnership, collaboration and support from: ELF and the ELF PAG, BRONCH-UK, the UK bronchiectasis registry and biobank, PROGNOSIS, the German Bronchiectasis Network, the Italian Society of Respiratory Medicine (SiMER), the US COPD Foundation, the Lung Foundation of Australia, the National Institute for Health Research UK, and the ECFSR.

\section{References}

1 Keistinen T, Savnajakangas O, Tuuponen T, et al. Bronchiectasis: an orphan disease with a poorly understood prognosis. Eur Respir J 1997; 10: 2784-2787.

2 Elborn JS. Bronchiectasis. In: Gibson GJ, Loddenkemper R, Sibille Y, et al., eds. The European Lung White Book. Sheffield, European Respiratory Society, 2013.

3 Chalmers JD, Aliberti S, Blasi F. Bronchiectasis in adults. Eur Respir J 2015; 45: 1446-1462.

4 Kelly MG, Murphy S, Elborn JS. Bronchiectasis in secondary care: a comprehensive profile of a neglected disease. Eur J Intern Med 2003; 14: 488-492.

5 Chalmers JD, Loebinger M, Aliberti S. Challenges in the development of new therapies for bronchiectasis. Curr Opin Pharmacother 2015; 16: 833-850.

6 Pasteur MC, Bilton D, Hill AT. British Thoracic Society guideline for non-CF bronchiectasis. Thorax 2010; 65: Suppl. 1, i1-i58.

7 Vendrell M, De Gracia J, Olverira C, et al. Diagnosis and treatment of bronchiectasis. Arch Bronconeumol 2008; 44: 629-640.

8 Chang AB, Bell SC, Torzillo PJ, et al. Chronic suppurative lung disease and bronchiectasis in children and adults in Australia and New Zealand Thoracic Society of Australia and New Zealand guidelines. Med J Aust 2015; 202 $21-23$.

9 Quint JK, Millett ERC, Joshi M, et al. Changes in the incidence, prevalence and mortality of bronchiectasis in the UK from 2004-2013: a population based cohort study. Eur Respir J 2015 [In press DOI: 10.1183/ 13993003.01033-2015].

10 Ringshausen FC, De Roux A, Diel R, et al. Bronchiectasis in Germany: a population based estimation of disease prevalence. Eur Respir J 2015 [In press DOI: 10.1183/13993003.00954-2015].

11 Seitz AE, Olivier KN, Adjemian J, et al. Trends in bronchiectasis among Medicare beneficiaries in the United States, 2000 to 2007. Chest 2012; 142: 432-439.

12 Roberts HJ, Hubbard R. Tends in bronchiectasis mortality in England and Wales. Respir Med 2010; 104: 981-985.

13 Ringshausen FC, de Roux A, Pletz MW, et al. Bronchiectasis-associated hospitalizations in Germany, 2005-2011: a population-based study of disease burden and trends. PLoS One 2013; 8: e71109. 
14 De Soyza A, Brown JS, Loebinger MR. Research priorities in bronchiectasis. Thorax 2013; 68: $695-696$.

15 Hurst JR. Microbial dysbiosis in bronchiectasis. Lancet Respir Med 2014; 2: 945-947.

16 Lonni S, Chalmers JD, Goeminne PC, et al. Etiology of non-cystic fibrosis bronchiectasis in adults and its relationship to severity. Ann Am Thorac Soc 2015 [In press DOI: 10.1513/AnnalsATS.201507-472OC].

17 Chalmers JD, Goemmine PC, Aliberti S, et al. The Bronchiectasis Severity Index: an international derivation and validation study. Am J Respir Crit Care Med 2014; 189: 576-585.

18 Haworth CS, Foweraker JE, Wilkinson P, et al. Inhaled colistin in patients with bronchiectasis and chronic Pseudomonas aeruginosa infection. Am J Respir Crit Care Med 2014; 189: 975-982.

19 Bilton D, Tino G, Barker AF, et al. Inhaled mannitol for non-cystic fibrosis bronchiectasis: a randomised, controlled trial. Thorax 2014; 69: 1073-1079.

20 Barker AF, O’Donnell AE, Flume P, et al. Aztreonam for inhalation solution in patients with non-cystic fibrosis bronchiectasis (AIR-BX1 and AIR-BX2): two randomised double-blind, placebo-controlled phase 3 trials. Lancet Respir Med 2014; 2: 738-749.

21 O'Donnell AE, Barker AF, Ilowite JS, et al. Treatment of idiopathic bronchiectasis with aerosolized recombinant human DNase I. rhDNase Study Group. Chest 1998; 113: 1329-1334.

22 EPIRARE. Guidelines for data sources and quality for RD Registries in Europe. https://web.archive.org/web/ 20160427135828/http://www.epirare.eu/_down/del/D4_GuidelinesfordatasourcesandqualityforRDRegistriesinEurope. pdf

23 Rare Diseases Task Force. Patient Registries in the Field of Rare Diseases: Overview of the issues surrounding the establishment, management, governance and financing of academic registries. www.eucerd.eu/?post_ type $=$ document $\& \mathrm{p}=1218$

24 Gatheral T, Kumar N, Sansom B, et al. COPD-related bronchiectasis: independent impact on disease course and outcomes. COPD 2014; 11: 605-614.

25 Jairam PM, van der Graaf Y, Lammers JW, et al. Incidental findings on chest CT imaging are associated with increased COPD exacerbations and mortality. Thorax 2015; 70: 725-731.

26 Winter DH, Manzini M, Salge JM, et al. Aging of the lungs in asymptomatic lifelong nonsmokers: findings on HRCT. Lung 2014; 193: 283-290.

27 Aksamit TR, Carretta E, Daley CL, et al. The Bronchiectasis Research Registry: a collaborative research cohort for non-cystic fibrosis bronchiectasis. Am J Respir Crit Care Med 2012; 185: A3654.

28 Quittner AL, O’Donnell AE, Salathe MA, et al. Quality of Life Questionnaire-Bronchiectasis: final psychometric analyses and determination of minimal important difference scores. Thorax 2015; 70: 12-20.

29 Murray MP, Pentland JL, Turnbull K, et al. Sputum colour: a useful clinical tool in non-cystic fibrosis bronchiectasis. Eur Respir J 2009; 34: 361-364.

30 Miller MR, Hankinson J, Brusasco V, et al. Standardisation of spirometry. Eur Respir J 2005; 26: 319-338.

31 Hurst JR, Elborn JS, De Soyza A. COPD-bronchiectasis overlap syndrome. Eur Respir J 2015; 45: 310-313.

32 Blasi F, Chalmers JD, Aliberti S. COPD and Bronchiectasis: phenotype, endotype or co-morbidity? COPD 2014; 11: 603-604.

33 Agusti A, Calverley PM, Celli B, et al. Characterisation of COPD heterogeneity in the ECLIPSE cohort. Respir Res 2010; 11: 122.

34 Chalmers JD, Smith MP, McHugh B, et al. Short and long term antibiotic therapy reduces airway and systemic inflammation in non-CF bronchiectasis. Am J Respir Crit Care Med 2012; 186: 657-665.

35 Finch S, McDonnell MJ, Abo-Leyah H, et al. A comprehensive analysis of the impact of Pseudomonas aeruginosa colonisation on prognosis in adult bronchiectasis. Ann Am Thorac Soc 2015; 12: 1602-1611.

36 Loebinger MR, Wells AU, Hansell DM, et al. Mortality in bronchiectasis: a long-term study assessing the factors influencing survival. Eur Respir J 2009; 34: 843-849.

37 McDonnell MJ, Jary HR, Perry A, et al. Non cystic fibrosis bronchiectasis: a longitudinal retrospective observational cohort study of Pseudomonas persistence and resistance. Respir Med 2014; 109: 716-726.

38 Reiff DB, Wells AU, Carr DH, et al. CT findings in bronchiectasis: limited value in distinguishing between idiopathic and specific types. AJR Am J Roentgenol 1995; 165: 261-267.

39 Winthrop KL, Aksamit TR, Olivier KN, et al. The respiratory microbiology of patients with nontuberculous mycobacteria from the United States Bronchiectasis Research Registry. Am J Respir Crit Care Med 2013; 187: A4541.

40 Chang AB, Brown N, Toombs M, et al. Lung disease in indigenous children. Paediatr Respir Rev 2014; 15: 325-332.

41 Chalmers JD, Hill AT. Mechanisms of immune dysfunction and bacterial persistence in non-cystic fibrosis bronchiectasis. Mol Immunol 2013; 55: 27-34.

42 Viviani L, Zolin A, Mehta A, et al. The European Cystic Fibrosis Society Patient Registry: valuable lessons learned on how to sustain a disease registry. Orphanet J Rare Dis 2014; 9: 81.

43 Tunney MM, Einarsson GG, Wei L, et al. Lung microbiota and bacterial abundance in patients with bronchiectasis when clinically stable and during exacerbation. Am J Respir Crit Care Med 2013; 187: 1118-1126.

44 Chalmers JD, McHugh BJ, Doherty C, et al. Mannose binding lectin deficiency and disease severity in non-cystic fibrosis bronchiectasis: a prospective study. Lancet Respir Med 2013; 1: 224-232.

45 Hill AT, Welham S, Reid K, et al. British Thoracic Society National Bronchiectasis Audit 2010 and 2011. Thorax 2012; 67: 928-930.

46 McShane PJ, Naureckas ET, Tino G, et al. Non-cystic fibrosis bronchiectasis. Am J Respir Crit Care Med 2013; 188: 647-656. 